\title{
Italique
}

Poésie italienne de la Renaissance

II | 1999

Varia

\section{Per un'ipotesi di lettura del Tasso autore del Mondo creato : la Divina Settimana di Ferrante Guisone}

Paola Cosentino

\section{(2) OpenEdition}

Edizione digitale

URL: https://journals.openedition.org/italique/203

DOI: 10.4000/italique.203

ISSN: $1663-4438$

Editore

Librairie Droz

\section{Edizione cartacea}

Data di pubblicazione: 1 décembre 1999

Paginazione: 143-165

ISBN: 2-600-00389-4

ISSN: 1423-3983

Notizia bibliografica digitale

Paola Cosentino, «Per un'ipotesi di lettura del Tasso autore del Mondo creato : la Divina Settimana di Ferrante Guisone», Italique [Online], II | 1999, online dal 07 octobre 2009, consultato il 24 novembre 2022. URL: http://journals.openedition.org/italique/203 ; DOI: https://doi.org/10.4000/italique.203 
PAOLA COSENTINO

PER UN'IPOTESI D I LETTURA DEL TASSO A U TORE

DEL MONDO CREATO:

LA DIVINA SETTIMANA

D I FERRA N T E G IS ONE 


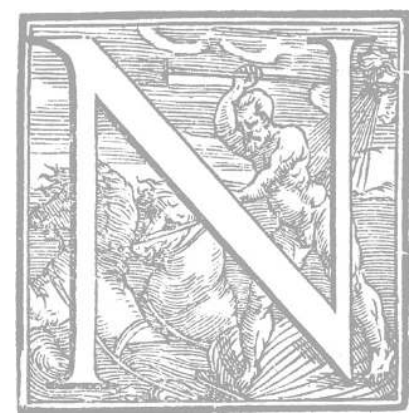

EL I592 appare a Tours, per i tipi di Giannetto Metaieri, la versione italiana della celebre Sepmaine del Du Bartas, tradotta in verso sciolto dall'ambasciatore mantovano Ferrante Guisone. ${ }^{\mathrm{I}}$ La dedica è rivolta al Serenissimo Signor Duca Vincenzo Gonzaga IV, duca di Mantova e di Monferrato: ${ }^{2}$ seguono una composizione latina in esametri, un'ode latina che porta la firma di Du Peyratius, ${ }^{3}$ un sonetto italiano di Gasparo Rienzo e uno francese di Roland du Jardin. Secondo questa edizione, la traduzione venne ultimata a Parigi il is agosto 1587.

Lavoro di grande qualità, già positivamente giudicato dal Croce, ${ }^{4}$ questa versione italiana del Guisone è soprattutto nota per una singolare coincidenza di date : il I592 è infatti l'anno in cui, almeno secondo il Manso, Torquato Tasso avrebbe cominciato a comporre Il Mondo Creato, poema in sciolti sulla creazione dell'universo, che sembrò collocarsi sulla scia del grande successo ottenuto dalla Sepmaine dubartasiana, con il quale l'eptamerone tassiano ha in comune le fonti classiche e patristiche.' Tasso conobbe il poema del Du Bartas, ed ebbe modo di leggerne una versione latina, probabilmente quella del Du Monin, ${ }^{6}$ pubblicata a Parigi già nel 1579 .

Sennonché, anche a tener nel debito conto quanto l'autore esplicitamente dichiara in proposito, e pur senza trascurare le questioni cronologiche, tutt'altro che chiare sotto il profilo della priorità, una lettura della Sepmaine dubartasiana attraverso la versione italiana di Ferrante Guisone pare molto istruttiva: il poema tassiano sembra presentare infatti significativi elementi di vicinanza con il poema del Du Bartas italianizzato dal Guisone. A questo scopo, ho proceduto a un confronto che non si limitasse ai riferimenti tematici, ma che mettesse in evidenza tratti linguistici e stilistici comuni alle due opere, dei quali si dà qui un primo saggio. Giova aggiungere questo nuovo tassello alla ricostruzione del catalogo di letture che contribuirono all'invenzione del poema tassiano, sulla cui genesi grava una quasi totale assenza di informazioni.

Di fatto, il lavoro del Tasso mantiene una sua autonomia creatrice più volte ribadita dalla polemica nei confronti dell'eptamerone dubartasiano, il cui ornatus troppo elaborato non è congeniale all'eloquenza misurata del poeta italiano. Del resto lo stesso Guisone aveva alleggerito lo stile troppo enfatico del modello francese, giungendo a una musicalità a tratti più vicina al poema tassiano. Il verso sciolto, utilizzato in chiave descrittivo-narrativa secondo il modello dell'esametro latino, accomuna $i$ due testi, li colloca su un medesimo piano che non a caso è quello della "traduzione " poetica, sovente realizzata 
in questo scorcio di secolo in endecasillabi non rimati. Da un lato, la versione italiana del poema dubartasiano, dall'altro, il nuovo De rerum natura cristiano : due poemi la cui istanza fondamentale è di farsi portatori di inoppugnabili verità di fede, dal momento che il genere didascalico diventa un efficace strumento attraverso il quale evocare l'origine divina del mondo.

Non debbono dunque stupire alcune singolari vicinanze fra le due opere, a cui dà particolare rilievo anche il confronto con $i$ dati storici e biografici in nostro possesso : alla considerazione fatta in esordio circa il Guisone, gentiluomo al servizio di Vincenzo Gonzaga, dobbiamo altresi aggiungere qualche nota sui legami che proprio con il duca di Mantova ebbe il Tasso. Nel settembre del I587 il poeta era, infatti, nella città lombarda, in occasione dell'incoronazione del duca: nuovamente a Mantova nel marzo del Is9I, avrebbe definitivamente lasciato la città, ripartendo alla volta di Roma, l'estate seguente. Guisone completò la sua traduzione nell'estate del I587 : l'avrebbe pubblicata nel I592. Verisimilmente, quell'anno Tasso aveva già posto mano al Mondo Creato.

L'esordio di entrambi i poemi ci offre immediatamente la cifra di una differenza ideologica e stilistica evidente : da una parte l'enfasi retorica tutta dubartasiana che celebra la potenza del divino creatore, ${ }^{7}$ dall'altra l'avvio tassiano, fortunata rielaborazione d'un verso petrarchesco (Rvf. LXII, I) dove è già racchiusa quella gravitas che sarà segno specifico dell'intero poema:

Padre del Cielo, e tu del Padre eterno

Eterno Figlio, e non creata prole,

De l'immutabil mente unico parto :

Divina imago, al tuo divino essempio

Eguale; e lume pur di lume ardente;

e tu, che d'ambo spiri, e d'ambo splendi,

O di gemina luce acceso Spirto,

Che sei pur sacro lume, e sacra fiamma,

Quasi lucido rivo in chiaro fonte,

E vera imago ancor di vera imago,

In cui se stessa il primo essempio agguaglia,

(Se dir conviensi) e triplicato sole,

Che l'alme accendi, e i puri ingegni illustri.

$$
(\text { Vv. I-I } 3)^{8}
$$

L'avvio è austero, solenne, caratterizzato da un procedere lento e uniforme, da una musicalità diffusa che trova il suo senso nell'uso sapiente dell'enjambement, nella spezzatura costante del verso : poema sapienziale, Il Mondo 
creato nasce da un'esigenza contemplativa e insieme conoscitiva, da un'istanza poetica ed esistenziale di autoannullamento che diventa consapevole omaggio a un Dio "immutabile» ed eterno. ${ }^{9}$ In questa epopea sulla creazione, Tasso sceglie di conciliare la tradizione platonico-ficiniana con un aristotelismo di matrice tomista : la Natura è presentata in costante movimento, sottoposta all'ordine perpetuo che regna su tutte le cose, immagine divina regolata da un'armonia che viene recuperata poeticamente attraverso il canto rivolto all'Altissimo. Il poeta è parte integrante di questa costru乏ione, è il mezzo attraverso il quale Dio può fare intendere la sua voce. A questo che è un topos allegorico di provenienza pitagorico-platonica si richiama un passo piuttosto noto della Prima Giornata del Mondo creato : si tratta della celebre invocazione a Dio, spirito che penetra l'universo e che, insieme, muove le corde di quell'umile strumento che è il poeta stesso: ${ }^{10}$

Signor, tu sei la mano, io son la cetra,

La qual, mossa da te, con dolci tempre

Di soave armonia, risuona, e molce

D'adamantino smalto i duri affetti.

Signor, tu sei lo spirto, io roca tromba

Son per me stesso a la tua gloria, e langue,

Se non m'ispiri tu, la voce e 'l suono.

Tu le tue maraviglie in me rimbomba,

Signore, e fia tua grazia il novo canto.

(vv. 63-7I)

Questo brano tassiano ba un suo corrispettivo nel poema di Du Bartas, ${ }^{11}$ allinterno del passo in cui il poeta francese tesse l'elogio dei cinque sensi attraverso un concreto riferimento alle componenti del corpo umano. Ai versi 592-96 della VI Giornata troviamo, infatti, descritte le proprietà della bocca :

Nostre langue est l'archet, nostre esprit le sonneur.

Nos dents, les nerfs batus, le creux de nos narines

Le creux de l'instrument, d'où ces odes divines

Prenent leur plus bel air, et d'un piteux accent

Desrobent peu à peu la foudre au Tout-pouissant.

Ma leggiamo la traduzione fornita da Ferrante Guisone, dove possiamo cogliere elementi che inducono a ipotizzare una relazione fra il poema tassiano $e$ la versione in endecasillabi dell'eptamerone francese: 
Noi per te risoniam di Dio la gloria :

La lingua è il pletro : il sonator lo spirto :

Sono i denti le corde : et è del naso

Il van quel de la citera, da cui

Prendon l'Ode divine il più bel canto :

E traggon con pietosi humili accenti

$\mathrm{Al}$ vero Giove i fulmini di mano.

Ricorre in entrambi i passi l'allusione alla "cetra", anche se i versi rimandano a contesti differenti, originati da una metafora dall'uno utilizzata in chiave mistico-esoterica, ${ }^{{ }^{12}}$ dall' altro risolta attraverso un riferimento simbolico a una precisa realtà musicale.

Il poema tassiano ci offre, in un suggestivo quadro dalle tinte allusivamente barocche, lo spettacolo della creazione, pervaso da una sublime malinconia, da un sentimento che deriva dalla mesta consapevolezza della caducità delle umane cose. Ma all'indiscutibile componente lirica che connota l'intero poema fa eco il messaggio dottrinale sotteso alla poesia ed esplicito nei versi relativi, in questa Prima Giornata, alla polemica nei confronti di Epicuro:

Quel, ch'i termini tolse al vasto mondo,

Le fiammeggianti mura a terra sparse,

E 'l vano immenso col pensier trascorse,

Non avria dato a Dea fallace ed orba

De la terra e del ciel lo scettro e 'l regno.

Folle, che non conobbe il modo e l'arte,

Per cui creato è il mondo al primo essempio,

Che 'l divino Architetto in sé dipinse,

Maggior de l'opra assai, che poscia offerse

Quasi da contemplar oggetto a' sensi.

(vv. 279-88)

Secondo Petrocchi, Tasso ha qui utilizzato l'elogio che Lucrezio rivolge proprio a Epicuro nel primo libro del De rerum natura (I, vv. 66-79). Il critico interpreta l'allusione a una "Dea fallace ed orba " come un implicito riferimento alla Ragione : eppure il contesto ci persuade a leggere in questa immagine una precisa evocazione della Fortuna. Come suggerisce Tagliaferri, "(il poeta) ha già parlato della teoria epicureo-lucreziana delle cause ai vv. 257-70, e attacca qui, al v. 282, quale "Dea fallace ed orba 》 non la Ragione, bensi la Fortuna, che agli occhi d'un cristiano doveva necessariamente apparire la motrice di un universo indifferente all'azione divina, sottoposto 
all'autonoma causalità meccanica delle aggregazioni atomiche e sottratto alle superiori volontà del creatore ». ${ }^{\mathrm{I}} \mathrm{Nel}$ poema dubartasiano troviamo una condanna decisa dell' atomismo democriteo che è ripresa dal Tasso : è istruttivo rileggerla nella traduzione del Guisone, poiché i versi contengono un richiamo diretto alla Fortuna, significativamente definita «fallace».

Quanto miri creò, non di Fortuna

Fallace man, qual vaneggiando finse

Democrito, cozzar facendo insieme

Con discorde union gli àtomi à caso.

Tout ce Tout fut basti, non des mains de Fortune

Faisant entrechoques par discordans accord

Du resveur Democrit' les invisibles corps.

(Du Bartas, I Jour, vv. I7-19) ${ }^{15}$

Se non è lecito affermare con certezza l'esistenza di una relazione fra $i$ testi, non possono però sfuggire talune palesi coincidenze nelle scelte lessicali $e$ contenutistiche. ${ }^{16}$ Confrontiamo, a questo proposito, $i$ versi che precedono il passo tassiano citato:

Ma queste (se dir lece) alte colonne

Ferma in ben salda base, e 'n lor s'appoggia,

Come a lui piace, la profonda terra ;

E crollar non la può tempesta o turbo.

(vv. 271-74)

Dio è presentato come sommo Artefice, come superno Creatore che si manifesta in tutta la sua potenza; è il « divino Architetto » che edifica il mondo e lo sottopone al suo volere, l'artista, ora pittore, ora scultore, che:

Come Dedalo, o Scopa, od altro antico

D'artificio gentil famoso mastro,

Prima raccoglie i peregrini marmi,

E i lucidi metalli, e i cedri eletti,

I quai del tempo e de l'età vetusta

L'invido dente non consumi e roda :

Poi forma il tutto, e la superba mole

Comparte e compie; e le sue volte, e gli archi

Fonda sovra marmoree alte colonne,

O pur di Caria a' simulacri appoggia.

(Secondo Giorno, vv. 63-72) 
In entrambi $i$ luoghi tassiani viene utilizzata la metafora delle "alte colonne ", secondo una concezione mitologica degli spazi celesti che, simili a un edificio, il Dio-artista va abbellendo di splendide forme. L'immagine è anche nel I Giorno dubartasiano:

Qual l'architetto di Real Palagio

Ch'anzi ch'a l'opra dar principio tenti,

Alta fabrica elegge, ove risplende

Con nobil gara et la ricchezza, et l'arte :

E tutte le beltà non ritrovando

In un solo edifitio accolte insieme :

De l'uno ei prende il frontespitio altero,

D'un altro le colonne, et l'ampie, e ricche

Scale d'un altro : et le piu rare cose.

[...] ainsi que fait le maistre

D'un bastiment royale, plus tost que de mettre

La main à la besogne, eslit un bastiment

Où la richesse et l'art luisent esgalement :

Et ne pouvant trouver en seul edifice

Toutes beautez en bloc, il prend le frontispice

De ce palais ici, d'un autre les piliers

D'un autre la façon des riches escaliers.

(I Jour, vv. I 83-90)

Il tema del mondo creato descritto come uno spettacolo in tutta la sua nascente magnificenza, come un "novo teatro" dove gli scranni degli spettatori sono ancora vuoti, poiché l'uomo non è ancora stato creato, è un'immagine che comunque appartiene al patrimonio culturale comune alle due opere, sebbene $i$ versi tassiani descrivano una realtà in fieri, percepibile attraverso la vaghezza che ancora la contraddistingue. La descrizione della materia prima, di quell'abbozzo informe e disorganico che costituisce il mondo delle origini s'ispira al testo biblico. Non è possibile cosi stabilire una diretta dipendenza di Tasso dal Du Bartas nei luoghi in cui i due poeti s'ingegnano a dipingere quel quadro affascinante che è la rappresentazione del nulla prima della creazione. ${ }^{17}$ Ma analizziamo la traduzione che Guisone fornisce dei versi del poeta francese : ${ }^{18}$

Il Ciel non risplendea di fiamme adorno,

Non spiravano odor le verdi piagge,

I pesci non fendean col nuoto l'onde,

Né de gli augelli gli amorosi canti 
Zèfir portava ancor su l'ale al Cielo :

Vedeasi il Tutto di bellezza ignudo,

Senza regola, et fiamma, et forma, et moto,

Et senz'alma : et non era foco il foco,

Il mar non era mar, l'aria non era

Aria, et non era ancor Terra la Terra

[...] Le ciel n'estoit orné

De grands touffes de feu : les plaines esmaillées

N'espandoyent leurs odeurs : les bandes escaillés

N'entrefendoyent les flots : des oiseaux les souspirs

N'estoient encore portez sur l'aille des Zephirs

Tout estoit sans beauté, sans reglement, sans flamme,

Tout estoit sans façon, sans mouvement, sans ame :

Le feu n'estoit point feu, la mer n'estoit point mer,

La terre n'estoit terre, et l'aer n'estoit point air

(I Jour, vv. 232-46)

La fitta serie di negazioni ricorrenti in questo brano preannuncia le bellezze non ancora esistenti di un mondo percepito nostalgicamente attraverso una serie di immagini che alludono a una primigenia età dell'oro : l'Eden futuro viene ritratto tramite un lungo elenco di immagini prefigurate, ma non ancora definite. Nel Tasso troviamo una costruzione analoga che sembra riecheggiare la traduzione guisoniana:

Non spiegavano ancor l'ombrosa chioma

Gli alberi eccelsi, e di lor fronde e d'ombra

Non facean vaga scena a' verdi colli.

Non fiorivano ancor rose e ligustri,

E i giacinti, e i narcisi, e gli altri fiori,

Né dipingeano il seno a' prati erbosi,

né fean lieta ghirlanda a' chiari fonti.

(vv. 396-402)

A proposito di questi endecasillabi, Petrocchi sottolinea la profonda differenza esistente fra Du Bartas e Tasso, differenza riscontrabile, secondo lo studioso, nei contenuti e nella diversa fattura poetica dei passi presi in esame. Eppure, nell'accostamento degli sciolti della traduzione e dei versi tassiani si riscontra un'identica impostazione ritmica, una medesima struttura retorica. Ancora, come nella versione dal francese troviamo : "Era una grossa bozza il Mondo in prima / una confusa mole, una sformata / mischianza ${ }^{19}$ (c. 6r), cosi nel Tasso leggiamo "Onde quella incomposta e rozza mole / Nè tutto era, ma nulla, e nulla apparve » (vv. 343-44). 
Celebre è poi il passo tassiano nel quale lo Spirito Santo viene rappresentato come un uccello intento a covare $i$ suoi piccoli. L'immagine è anche nel Primo Giorno dubartasiano: Petrocchi smentisce l'ipotesi di vicinanza dei due testi, limitandosi a ribadire che la metafora della covata è presente in molti scritti dei Padri della Chiesa. Ma è bene rileggere i testi. Scrive Tasso:

Anch'ei presente a l'opra

Spirando gì forza e virtute a l'onda,

D'uccello in guisa, che da frale scorza

Col suo caldo vital covata e piena,

Trae non pennato il figlio, e quasi informe.

(vv. $528-32$ )

Nel suo studio dedicato alla ricerca delle fonti del poema tassiano, ${ }^{20}$ Proto ammette, a proposito di questi versi, che "l'immagine dello spirito covante è in S. Basilio : ma questi, come rilevo da una citazione di S. Tommaso (I, LXXIV, 3) dice - id est, fovebat et vivificabat naturam aquae ad similitudinem gallinae incubantis... - : mentre il T. parla di un uccello in generale, che cova i figli, avvicinandosi più specialmente al Du Bartas. Che la similitudine derivi dallo scrittore nascosto in quella strana citazione?». Una risposta affermativa a questo interrogativo può derivare dalla versione che, degli alessandrini dubartasiani, fornisce Ferrante Guisone :

[...] o qual l'augello

Ch'a l'uova proprie, overo a l'adottate

Cerca dar vita, sopra vi si corca,

Et col proprio calor d'un giallobianco

Rotondo un gaio pulcinello aviva.

Ou bien comme l'oiseau qui tasche rendre vifs

Et ses œufs naturels, et ses œufs adoptifs,

Se tient couché sur eux, et d'un chaleur vive,

Fait qu'un ronde jaune-blanc en un poulet s'avive.

(I Jour, vv. 297-300)

Il confronto operato fra il Primo Giorno del poema tassiano e la traduqione guisoniana della Sepmaine di Du Bartas ha messo in rilievo significative sourapposizioni, che offrono, se non altro, la prova di un percorso affine, orientato nella direzione di una lingua poetica a tratti molto simile. Una rapida ricognizione delle restanti sei Giornate, passibile di ulteriori notazioni da fornire in altra sede, non fa che confermare quest'assunto, a partire dal quale si puo arrivare a sottolineare l'importanza del ruolo svolto dalla ver- 
sione del Guisone. Nella Seconda Giornata dell'eptamerone tassiano, troviamo illustrate le singole componenti dello Zodiaco, corrispondenti alla descrizione che della volta stellata Du Bartas fornisce nel Quarto Giorno. Tasso si rifà agli Aratea di Cicerone: pur sottolineando polemicamente il carattere menzognero delle antiche favole greche, il poeta non rinuncia a servirsene nella raffigurazione delle costellazioni. ${ }^{21}$ Questi i versi tassiani:

La Vergine vicina a lui risplende

Con l'aurea spiga : e poi la luce e l'ombra

L'alta Libra celeste agguaglia in lance.

(vv. 359-6I)

Nella versione guisoniana sono da notare la rispondenza significativa dei termini impiegati, sebbene il dettato poetico sia qui volto a rendere la magniloquenza degli alessandrini francesi :

Lunge non è la Vèrgine, che sgombra

Dal Ciel le nubi col dorato manto,

Che ne la destra l'ale, e 'n la sinistra

La spica tiene alteramente ùmile

Dopo la Vèrgin la Bilancia segue,

Che la notte egualmente, e 'l giorno libra:

D'oro son le sue lanci, e 'i sei cordoni.

La Vierge n'est pas loin, qui du train flamboyant

De son doré manteau le bleu Ciel baloyant

Porte d'une façon humainement superbe

Des ailes en la dextre, en la gauche une gerbe.

Apres les feux puceaux le Trebuschet reluit,

Qui justement balance et le jour et la nuict :

D'or sont ses deux bassins, ses six cordons sont d'or.

(IV Jour, vv. 23 I-37)

Il III Giorno del Mondo Creato si apre con l'evocazione di quei diletti mondani che S. Basilio aveva condannato nella quarta delle sue omelie: la rappresentazione di una civiltà antica ormai scomparsa conferisce ai versi un fascino sottilmente legato al vagheggiamento malinconico di lontane abitudini cortigiane definitivamente abbandonate. Il carattere particolare di questa che è una vera e propria introduzione lirica posta all'inizio del Terzo Giorno richiama gli esordi delle altre Giornate, dove l'autore si concede una pausa riflessiva, uno spazio di poetica affabulazione. Ritratto elegiaco di passati 
splendori, Tasso dà vita, in questi pochi versi (vv. I-43), a un quadro vivace in cui sono raffigurati i piaceri della convivialità celebrati nell' allusione ai giochi, alla musica, alle rappresentazioni teatrali : rapido, lo sguardo del poeta si volge poi alla contemplazione del Creato, dagli « stellanti chiostri » alla "gravissima, vasta e rozza terra ». Nel descrivere la creazione delle acque, Tasso si sofferma a narrare $i$ movimenti delle maree determinati dalle fasi lunari, esemplando $i$ suoi versi sulle osservazioni contenute nella Naturalis Historia di Plinio :

Altri mari vi son, come s'afferma, Che ne lo spazio pur d'un mese integro

Soglion due volte alzar l'onde spumose,

$\mathrm{E}$ due volte inchinarle in sé ripresse.

Anzi nel mar de gli Etiopi adusti

Non v'ha flusso e reflusso.

(vv. 200-205)

Altri esempi fornisce poi l'autore per documentare le diverse turbolenze dei mari :

Ma 'l Ligustico seno, e quel de' Toschi, Ch'ondeggia appresso a la novella Pisa, Ch' a più onorati studi i premi serba, E le corone a le più dotte fronti,

Non ha quasi de l'onde il moto alterno.

(vv. 21 8-22)

Nel III Giorno della Divina Settimana, dove si fa riferimento alle mutevoli condizioni delle acque, troviamo:

Ma, che più è, si vede il mar d'Atlante

Piu surmontare i lidi, che lo stretto

Di Bòsforo, o il Ligùstico non face :

Et che flusso, o riflusso unqua non hanno

Le paludi, che son figlie del mare.

Qui plus est, nous voyons que la mer Atlantique

Se desborde plus loin, que ni la Ligustique,

$\mathrm{Ni}$ la Bosphoriene, et qu'encor les palus,

Qui naissent de la mer, n'ont ni flus ni reflus.

(III Jour, vv. I 89-92) 
La presenza di identiche soluzioni lessicali nello spazio di pochi versi (il "flusso e reflusso» dei mari, cui si allude per negazione nel riferimento alle paludi; il "Ligustico» mare che nel Tasso diventa "Ligustico seno", che trovano peraltro una perfetta corrispondenza nell'originale francese) è forse indizio di una contaminazione involontaria, conseguente a una lettura rapidamente assimilata. Meglio si prestano a questa ipotesi i seguenti versi, che, pur facendo parte dell'ampia digressione sui movimenti che regolano le acque, offrono al poeta una nuova occasione di lode al Creatore:

Anzi fu l'alta man del Mastro eterno,

Che 'n tante forme figurollo e finse,

Or facendo il mar lungo, or tondo, or quadro,

E'n guisa di piramide e di croce

Anco formollo, e di mirabil vaso.

(vv. 283-87)

Thibaut de Masières evidenzia la somiglianza riscontrabile fra $i$ versi tassiani e il passo del III Giorno dubartasiano riferito alla creazione delle acque ${ }^{23}$ :

Dieu respandit les flots sur la terre feconde,

En figure quarree, oblique, large, ronde,

En pyramide, en croix, pour au milieu de l'eau

Rendre nostre Univers et plus riche et plus beau.

(vv. 77-80)

Più chiaro risulterà il legame fra le due opere, se prendiamo in esame la versione guisoniana dei versi francesi :

De' suoi ruscelli quasi a un tempo stesso

Sopra l'unita tavola formando

Ogni sorte di forma : in su 'l terreno

Dio così l'onde con figura sparse

Quadra, sferica, obliqua, ampia : et altrove

Conforma di piràmide, o di croce.

"Ce qui compte ici» sostiene Erba ${ }^{24}$ "ce n'est pas tant la similarité du topos que le choix des images tout à fait caractéristiques et, pourrait-on dire, portant un sceau d'authenticité, presque une signature. Selon Petrocchi, la source commune dans ce cas est Strabon, mais ces références précises et originales à la géométrie plane et à la géométrie dans l'espace sont de Du Bartas 
avant d'être du Tasse». L'immagine delle acque modellate secondo diverse forme sembra essere passata invariata dal testo francese a quello italiano, probabilmente attraverso la mediazione guisoniana: la dipendenza diretta dei versi da una fonte comune non inficia la prova evidente di talune identità terminologiche e metriche rintracciabili all'interno dei brani presi in esame. A un diretto rapporto con il poema del Du Bartas si richiama, poi, una divagazione tassiana che si colloca sulla scia della polemica nei confronti di Epicuro già tratteggiata nel Primo Giorno ${ }^{25}$ :

Ma sol il peregrino ed alto ingegno

Ch' ascende al cielo, e gli stellanti chiostri

Di spera in spera alfin trapassa e varca

I confini del mondo, e i spazi angusti

Esposti a' sensi, e con eterna pace

$\mathrm{Si}$ congiunge a le pure eterne menti.

(vv. 624-29)

Quel "peregrino e alto ingegno » che « di spera in spera alfin trapassa e varca $i$ confini del mondo» corrisponde allimmagine dello spirito umano che "s'eslance dehors / Les murs de l'Univers... " (VI Jour, vv. 808-809; trad. : "De l'Universo oltre i confin si scaglia ", c. Izov) e che si congiunge " a le pure eterne menti», dal Du Bartas chiamate "courtisans celestes》 (1vi, v. 8II; trad.: "Spirti Angelici beati», c. I3ov).

Dedicato al racconto della creazione del sole e della luna, il Quarto Giorno è modellato, a tratti quasi pedissequamente, sulla VI Omelia di S. Basilio: l'intento dottrinale lascia spazio, nel Tasso, al vagheggiamento poetico degli astri, all'effondersi lirico di un sentimento che è contemplazione e insieme percezione commossa della presenza divina nel cosmo.

Perchè dar volle in cielo un chiaro essempio

Col variar de l'incostante luna

A l'incostanza umana, al modo incerto

Di nostra vita instabile e vagante,

Ch' un perpetuo tenor giamai non serba

Né 'n fermo stato si mantiene e dura.

(vv. 402-407)

Sono gli endecasillabi in cui il poeta paragona le trasformazioni del satellite terrestre alle variazioni cui è sottoposta la vita umana, dominata dallincostanza e dal mutevole aspetto d'ogni passione e di ogni affetto. E lo stesso tema è al centro del canto intonato alla Luna nella Quarta Giornata della creazione dubartasiana: 
La Divina Settimana di Ferrante Guisone

O vaga Cinthia, de' Celesti lumi

Secondo honor, de le divine feste

Secura norma, guida a' peregrini,

Del mar Reina, che 'l riposo brami,

E 'l sonno placidissimo ne adduci :

Che dirò io de l'argentate corna,

Et de la fronte tua varia, e incostante ?

Che 'l mio spirto, hor da questo, hor da quella

Bilanciando, sospende, e in dubbio tiene?

O le second honneur des celestes chandelles, Asseuré calendrier des Fastes eternelles,

Princesse de la mer, flambeau guide-passant,

Conduy-somme, aime-paix ! que diray je, o Croissant,

De ton front inconstant, qui fait que je balance,

Tantost çà tantost là d'une vaine inconstance?

(IV Jour, vv. 648-52)

Con l'espressione "argentate corna», Guisone rende il francese "le croissant", termine che Du Bartas sovente utiliza per designare il satellite terrestre. In un notturno bellissimo, Tasso fa allusione alle influenze lunari, adoperando quel sintagma (" argentee corna », v. 76) che già si è visto impiegato nella tradurione guisoniana:

Però, quando ella col suo cerchio intero

Mostra da l'alto cielo il pieno aspetto,

Emula vaga del fratello ardente,

$\mathrm{E}$ (se dir lece) quasi un sol notturno,

Allor le notti tiepide e serene

Son più de l'altre, in cui d'adunca falce

Mostra l'imago, o con argentee corna

S'incurva avanti il sole, o pur da tergo.

Allor via più germoglia il verde tronco

Con nove frondi e rami, e più s'impingua

L'umida sua midolla entro la scorza.

(vv. 470-80)

La contemplazione dei corpi celesti genera una poesia dell'idillio che caratterizza numerosi luoghi del Mondo Creato : all'interno di un percorso narrativo definito dai tempi della creazione, possono infatti individuarsi singoli momenti descrittivi, scorci lirici arricchiti dal fascino che emana una Natura colta nel suo primo, aurorale apparire. ${ }^{26}$ Altrettanto seducenti sono $i$ 
versi che Du Bartas dedica agli influssi della Luna, che nella versione guisoniana creano una sorta di suggestiva eco alla poesia degli sciolti tassiani:

Taccio che Nereo copre, e scopre i lidi

Per lo cangiato aspetto de la Luna :

Che quanto ne' viaggi suoi notturni

Ella più cresce, piú de gli animali

Ne le dure ossa le midolla cresce :

Il sangue ne le vene : et ne le piante

L'humor : ne l'ostree la bavosa carne

J'oublieray que la mer s'enfle et se diminue

Par l'acroist et decroist de l'estoille cornue :

Que tant plus elle croist en ses nuicteux travaux,

Tant plus croist la mouelle es os des animaux,

Dans les veines le sang, la seve dans les plantes,

Et la baveuse chair dans les huitres flotantes

(IV Jour, vv. 435-40)

Il V Giorno tassiano si apre con una similitudine che sintetizza poeticamente l'argomento del canto. L'uomo è paragonato a un pellegrino che, in procinto di lasciare la casa dove è stato accolto, si volge a ringraziare $i$ suoi ospiti : nel momento in cui, chiamati a far ritorno in cielo, abbandoneremo la terra, riconoscenti dovremo rivolgere lo sguardo a questa «opaca chiostra» che ci ha nutrito e curato come suoi figli. La terra, con le sue muliebri forme, è la protagonista di questa Giornata del Mondo Creato, dove il poeta indulge a patetiche quanto struggenti descrizioni di una Natura straordinariamente ricca di forme e di colori, rappresentata come madre benigna e amica.

Troviamo qui la lunga digressione sulla fenice, poeticamente costruita a partire dalla traduzione del De ave phoenice dello pseudo-Lattanzio, come si documenta nell'importante saggio di Bruno Basile, ${ }^{27}$ che ha analizzato $i$ meccanismi compositivi tassiani all' interno della trattazione poetica della creazione divina degli animali. Tasso interpreta il mito della fenice in chiave allegorica, facendone l'emblema della resurrezione di Cristo, secondo quanto sembra suggerire un passo tratto dall' Hexameron di S. Ambrogio che allude alla renovatio carnis, simboleggiata dal perenne rinascere del " felice angello ». Questa sourapposiżione è anche in Du Bartas, il quale, facendo ricorso al poemetto pseudo-lattanziano, non trascura la suggestione ambrosiana, attraverso cui la leggenda della fenice può essere riletta come allegoria religiosa. E, a questo proposito, Basile aggiunge che "tra l'episodio della «celeste Phoenix 》 del poeta ugonotto francese e la Fenice del Tasso non esiste alcun rapporto stilistico documentabile, ma è un dato di fatto assolutamente 
certo che i due poeti, a distanza di tempo e di riferimenti culturali, si piegarono a qualcosa di curiosamente analogo [...] Quello che mette conto notare in questa sede è, accanto al manierismo del francese, la sua disponibilità, identica a quella tassesca, a rinvenire nel mito tracce cristologiche $\gg .{ }^{28}$

Nelle due ultime Giornate consacrate alla creazione del mondo vengono rispettivamente evocate dal Tasso le caratteristiche degli animali che popolano la Terra (VI Giorno) e la comparsa dell'nomo nel Paradiso terrestre ${ }^{29}$ (VII Giorno). Passando velocemente in rassegna i versi, è risultato evidente il legame, ancora una volta attestabile attraverso il diretto raffronto dei passi, fra alcuni sciolti tassiani e la traduzione guisoniana. Toldo ${ }^{30}$ aveva già sostenuto, senza tenere conto però delle fonti comuni ai due testi, che la descrizione fisica dell'uomo risultava costruita secondo un medesimo schema, evidentemente ripreso dal Tasso su ispirazione del Du Bartas. Petrocchi, nel suo commento al Mondo Creato, ritiene invece che i versi dedicati alla creazione del primo uomo siano, in realtà, modellati sul De hominis structura dello pseudoBasilio : non può però sfuggire la marcata vicinanza di taluni modi poetici, frutto, forse, di una conoscenza non rivelata, di una reminiscenza inconsapevole della celebre tradurione della Sepmaine.

Nella posizione eretta dell'nomo, i cui occhi sono rivolti al cielo a differenza di tutte le altre specie animali, non dobbiamo trovare, suggerisce il poeta, motivo di superbia:

Così il Fabro primier la fronte e gli occhi

Alzò de l'uomo a le stellanti spere,

Perché là guardi, onde celeste origo

Ebbe l'alma immortal, ch'eterno regno

Par che chieda per grazia al Padre eterno.

(VII Giorno, vv. 5 I I-1 5)

Alla contemplazione del proprio luogo d'origine cui l'anima guarda attraverso gli occhi, fanno riferimento $i$ seguenti versi della Divina Settimana :

Non gli piegasti verso il centro il viso :

Anzi le luci inver le sante fiamme

Tu gli inalzasti de l'immenso Cielo,

Onde ad ogn'hor la sua origo altera

L'alma contempli, aventurosa, il loco.

Ne courbant toutes fois sa face vers le centre

Comme à tant d'animaux, qui n'ont soin que du ventre 


\section{Paola Cosentino}

Mourant d'ame et de corps : ains relevant ses yeux

Vers le donnez flambeaux qui brillent dans les cieux.

(VI Jour, vv. 493-96)

La «celeste origo 》 (v. 5I3) come variante di «origo altera 》 sembra individuare un nesso, un rapporto fra $i$ due testi : più avanti, l'illustrazione della disposizione dei sensi, sapientemente ordinati come " sentinelle » protettrici del corpo, consente un altro interessante accostamento. Leggiamo gli sciolti tassiani:

\footnotetext{
Ma pur sì come in rocca 'en torre eccelsa Son disposte le guardie intorno intorno. Onde sicura da notturna insidia

Il nemico lontan si scopre e vede;

Così a guardia i veloci e desti sensi

Collocò nella testa il Fabro eterno.
}

(vv. 56I-66)

Questa, invece, la descrizione della vista e dell'udito fornita da GuisoneDu Bartas :

Gli occhi, Duci del corpo, à guardìa posti

Di questa umana Rocca in alto furo

Les yeux, guides du corps, sont mis en sentinelle

$\mathrm{Au}$ plus notable endroit de ceste citadelle

(VI Jour, vv. 509-10)

Orecchie, uscio de l'alma, et sentinelle

di questo corpo?

Qu'es conduits tortueux des jumelles oreilles,

Portieres de l'esprit, escoutes de nos corps

(ibidem, vv. 598-99)

Con il termine "rocca», Guisone traduce l'espressione dubartasiana "citadelle », dimora dell'intelletto umano (" bumain entendement» per il poeta francese) o più precisamente dell'anima, come Tasso afferma seguendo la dottrina platonica:

Ne l'alta adunque de la nobil testa

Rocca fondolla, e quasi in propria reggia. 
Ivi de l'uom, ch'è quasi un picciol mondo, ${ }^{32}$ A lei concesse l'onorato impero.

Alla descrizione di un Eden primigenio dove Adamo si muove innocente e padrone del mondo, succede il racconto della creazione di Eva, ${ }^{33}$ evocata attraverso pochi, rapidi accenni conformi alla narrazione biblica: sono i versi conclusivi di un excursus di contemplazione e di speculazione dottrinale che, in quest'ultima parte, sembrano rinunciare all'erudizione per farsi canto, preghiera, inno sacro. Tasso forgia una sorta di testamento morale che, se da una parte rivela una vena poetica ancora non del tutto esaurita, dall'altra mostra una straordinaria capacità di affrancamento da ogni orpello retorico, tale da permettere al verso di aderire alle radici stesse del sentimento religioso. Riscrittura sapiente dei testi sacri, revisione in chiave cattolica del poema lucreziano sulla natura, reinterpretazione mistica del poema didascalico cinquecentesco, il Mondo Creato mostra di avere un profondo legame sia con la tradizione patristica riscoperta dalla filologia umanistica, sia con l'ambiente dove ebbe ampia diffusione la Sepmaine ou création du monde del Du Bartas : ${ }^{34}$ il poema della creazione del mondo passa dalla Francia all'Italia attraverso la versione in endecasillabi sciolti di Ferrante Guisone e quali suggestioni ne abbia ricavato il Tasso per il suo poema si è voluto chiarire nel corso di questo scritto. Quanto abbiamo fin qui argomentato vuole offrire, infatti, nuovi elementi per documentare una lettura del Du Bartas compiuta dall'autore del Mondo Creato attraverso una traduzione che risulta non priva di una sua consistenza poetica.

Paola Cosentino 


\section{Paola Cosentino}

I. Scarse e frammentarie sono le notizie in nostro possesso su Ferrante Guisone (o Guisoni) : ambasciatore al servizio di Vincenzo Gonzaga, curò la pubblicazione dell' Aminta di Torquato Tasso in Francia nel 1584. Cfr. J. Balsamo, Les Rencontres des Muses, Genève, Slatkine, I992, pp. 279-80.

2. LA | DIVINA | SETTIMANA : | TRADOTTA DI | RIMA FRANCESE IN | VERSO SCIOLTO | Italiano | In TOURS | Appresso Giannetto Metaieri, | Regio Stampatore | MDXCII | CON PRIVILEGIO | In I 2, [VI] - I 52 . Due esemplari di quest'edizione (cui G. Resta fa riferimento in una nota del suo articolo Una lettera inedita del Tasso e il "Mondo Creato", in «Convivium », N. S., 25, I, I957, pp. 77-82) si trovano alla Bibliothèque Mazarine a Parigi (n. 22066-n. 22067). A proposito delle edizioni italiane della Divina Settimana, F. Tomasi, sulla scorta del Resta, nota che l'edizione in sedicesimo del Mondo Creato, pubblicata integralmente dal Ciotti a Venezia nel i608, presenta le medesime incisioni apparse sulle ristampe della traduzione guisoniana edite, sempre presso il Ciotti, a partire dal I 593 (vedi «La malagevolezza delle stampe». Per una storia dell'edizione Discepolo del "Mondo Creato", in «Studi Tassiani», 42, I994, pp. 43-78, a p. 53 nota).

3. Per quanto riguarda il Du Peyratius (latinizzazione del francese Du Peyrat), si vedano le indicazioni di H. Vaganay, Un français «italianisant» peu connu, extrait de la «Revue du Seizième Siècle ", I6, I929.

4. Cfr. B. Croce, Intorno a Guglielmo Du Bartas, in Nuovi saggi sulla letteratura italiana del Seicento, Bari, Laterza, $1968^{3}$, p. 208 nota.

5. G. Petrocchi, curatore dell'edizione del poema tassiano pubblicata nel i95 I, dimostra agevolmente come talune vincinanze fra Du Bartas e Tasso siano da attribuirsi all'utilizzo delle medesime fonti. Gli esameroni di S. Basilio e di S. Ambrogio sono, infatti, alla base delle riscritture cinquecentesche del Genesi, come lo sono le fonti classiche quali il De caelo et mundo di Aristotele e il De rerum natura di Lucrezio.

6. G. Resta cit., presenta una lettera inedita del Tasso, indirizzata a Maurizio Cataneo, che risale alla fine del settembre I 594 : alludendo alla stesura del Mondo Creato, il poeta scrive : «Piaccia a Dio di concedermi tanto de vita che basti a questa opera almeno, la quale io scrivo in verso sciolto toscano, perchè di scriverla in verso esametro latino da l'infirmità non mi è conceduto. So ch'è stata scritta da Francesi : ma il verso latino non mi piace, del francese non fo alcun fermo giudizio... ». Secondo G. Jori (in Le forme della creazione. Sulla fortuna del « Mondo creato» (Sec. XVII e XVIII), Firenze, Olschki, I995), Tasso qui sottolinea esplicitamente la sua distanza dall'opera dubartasiana nonché dalla traduzione in esametri latini, entrambe formalmente lontane dal gusto e dalla sublime compostezza degli endecasillabi del Mondo Creato. È comunque opportuno precisare che nel 1583 fu pubblicata a Parigi un'altra versione latina della Sepmaine dubartasiana a cura di Gabriel de Lerme : potrebbe essere questa l'opera a cui fa riferimento il Tasso, piuttosto che quella del Du Monin : lo suggerisce P. Luparia in una nota del suo $I l$ « Mondo Creato » poema sapienziale, in «Giornale storico della letteratura italiana», I64, I987, pp. I-33. Sul volume di Jori si veda la recensione di V. De Maldé, in «Studi Tassiani», 44, I 996, pp. $250-55$.

7. Du Bartas intona un canto sostenuto, magniloquente, ricco di metafore che fanno spesso ricorso alla mitologia : «Toy qui guides le cours du ciel porte-flambeaux / Qui, vray Neptune, tiens le moite frein des eaux, / Qui fais trembler la terre, et de qui la parole / Serre et lasche la bride aux postillons d'Aeole » (Dal Premier Jour della Sepmaine ou creation du monde di Guillaume Saluste Du Bartas, éd. Y. Bellenger, Paris, Société des Textes Français modernes, 1994, vv. I-4), che il Guisone così traduce : "Signor, che volgi il fiammeggiante Cielo : / Et del grande Oceàn freni l'orgoglio, / Netunno vero : et l'ampia Terra scuoti / Serrando a un cenno, et disserrando i venti » (La Divina Settimana, ed. cit., c. Ir). 


\section{La Divina Settimana di Ferrante Guisone}

8. Cfr. T. Tasso, Il Mondo Creato, a cura di G. Petrocchi, Firenze, Le Monnier, I95 I, p. 3.

9. L'esordio del Mondo Creato rivela una robusta tessitura retorica, che si incentra su di un raffinato utilizzo delle perifrasi, dove, a differenza del Du Bartas, Tasso tende a mettere in evidenza il solido apparato dottrinale dal quale prendono spunto i suoi versi.

Io. Metafora platonica arricchita dagli accenti dolenti di un canto che in sé racchiude molteplici allusioni di derivazione orfica e gnostica, il passo tassiano fa altresì riferimento alla letteratura patristica. I versi sembrano offrire spazio a una nuova concezione della poesia, che Tasso considera strumento di rivelazione divina, di affermazione di nuove verità. Secondo G. Scianatico (cfr. Ilpoema « meraviglioso ». Per un'ipotesi sul «Mondo Creato», in Dall'idillio alla visione, Manduria-Bari-Roma, Lacaita, 1996, pp. 6r-99), gli sciolti tassiani indicano, insieme ai contemporanei Discorsi sul poema eroico, al Giudizio sopra la Gerusalemme e alle Esposizioni aggiunte alle Rime nel i 593, « una svolta progressiva di poetica non circoscritta nell'ambito letterario, che si radica beninteso nel sentimento di insecuritas che inquieta la concezione del mondo alla fine del Cinquecento » (p. 69).

I I. Cfr. G. Jori cit., p. 69 e segg. : «Il Du Bartas paragona l'organo della parola a uno strumento musicale suonato da Dio. Concetto che ha qualche somiglianza con la dichiarazione di poetica del Mondo creato più volte citata, dove il Tasso introduce le metafore della "roca tromba" e della "cetra" ".

I 2. A questo proposito, cfr. B. Basile, La cetra «discorde» del Tasso, in «Lettere italiane», 37 , I985, pp. 493-500 e E. Ardissino, "L'aspra tragedia ». Poesia e sacro in Torquato Tasso, Firenze, Olschki, 1996, pp. 49-52.

13. Cfr. P. Tagliaferri, $I l$ «Mondo creato» di Torquato Tasso tra letteratura e teologia, in «Studi italiani », 6, I, 1994, p. 75. Una lunga e approfondita dissertazione in merito al legame esistente fra il Mondo Creato e il De rerum natura è contenuta nel volume di B. Basile dedicato alla produzione poetica dell'ultimo Tasso (Poëta melancholicus. Tradiz̨one classica e follia nell'ultimo Tasso, Pisa, Pacini editore, 1984) : nel saggio Follia e ragione : Tasso lettore di Lucrezio, lo studioso svolge un attento esame dell'edizione del poema lucreziano annotata dal Tasso e appartenente al Fondo Barberiniano presso la Biblioteca Apostolica Vaticana. Ancora sul Mondo Creato e il De rerum natura, si veda M. Saccenti, Lucrezio in Toscana. Studio su Alessandro Marchetti, Firenze, Olschki, I 966.

I4. B. Basile nota che il tema lucreziano viene riproposto in chiave polemica anche nella Sepmaine : «Eschelle qui voudra les estages des cieux, / Franchisse qui voudra d'un saut ambiteux / Les murs de l'univers, et, bouffi d'arrogance, / Contemple du grand Dieu face à face l'essence » (I Jour, vv. I05-I09), che Guisone traduce « Poggi dunque chi vuol di Cielo, in Cielo, / et surmonti le mura, ambizioso, / De l'universo : et, gonfio d'arroganza, / Contempli in faccia il sommo eterno Dio » (c. $3 \mathrm{v})$.

I s. A partire da questa citazione, saranno forniti, con gli sciolti della traduzione, i corrispondenti alessandrini di Du Bartas.

I6. Al dibattito riguardante il legame fra Du Bartas e Tasso, ha aggiunto il suo contributo L. Erba, Quelques remarques à propos de "La Sepmaine» et du «Mondo Creato», in Du Bartas, poète encyclopédique du XVI siècle, Lyon, La Manifacture, I 988, pp. I 3 I-40. Allo scopo di dimostrare la sostanziale differenza fra i due poemi, che si evidenzia nell'appartenenza dell'uno, la Sepmaine, a una maniera ancora tutta cinquecentesca, e dell'altro, Il Mondo Creato, a un barocco che prefigura il Seicento, Erba dimostra come talune somiglianze fra i due testi possano essere attribuite a un curioso fenomeno di «poligenesi» letteraria, ascrivibile al comune repertorio di fonti, nonché al contesto storico e culturale cui le due opere appartengono. 


\section{Paola Cosentino}

17. L. Erba cit., sostiene che la differenza fra i due testi si rileva soprattutto dall'opposta maniera poetica che essi utilizzano per rappresentare una realtà non ancora definibile come tale : mentre Du Bartas descrive un mondo in formazione, Tasso ne definisce i tratti attraverso una serie di negazioni, che mostrano «un paysage peuplé d'absences qui évoquent un site idyllique, voire édenique» (p. I39).

18. Secondo Jori, Du Bartas forgia i suoi versi attraverso « un groviglio di allitterazioni, annominationes, antitesi e iperboli che in parte si affidano alla cosmogonia di un autore classico, Ovidio » (G. Jori cit., p. 17) ; al contrario, Tasso «adoperò anche qui, probabilmente non senza polemica nei confronti de Du Bartas e di quel rigoglio di immagini, la propria caratteristica misura » (ibidem, p. I 8). Del resto, diversa sembra essere anche l'interpretazione dottrinale del passo del Genesi sull'origine della creazione.

I9. Questi i versi corrispondenti del Du Bartas : « Ce premier monde estoit une forme sans forme / Une pile confuse, un meslange difforme » (I Jour, vv. 223-27). Al v. 467, il dubartasiano «masse flottante» è tradotto da Guisone «imperfetta mole ».

20. Cfr. E. Proto, Per le fonti del «Mondo Creato», in «Rassegna critica della letteratura italiana ", I 4, I909, pp. 193-235.

21. Nel V Giorno, il giudizio negativo del poeta e l'esplicita rinuncia a trattare della favola di Arione (vv. 244 e segg.) sembrano fare una polemica allusione alla Sepmaine dubartasiana, dove una lunga digressione è dedicata alla rievocazione del celebre mito.

22. Entrambi i poemi si richiamano a un passo del I libro delle Georgiche, quando Virgilio, alludendo alla costellazione della Bilancia, dice : «Libra dies somnique pares ubi fecerit horas / et medium luci atque umbris jam dividit orbem» (vv. 208-209).

23. «C'est un assemblage de qualificatifs curieux, qui ne sont exigés ni par l'idée exprimée, ni par le sujet traité; on a chance d'en chercher vainement l'origine dans une source commune» (M. Thibaut de Masières, Les poèmes inspirés du début de la Genèse à l'époque de la Renaissance, Louvain, Uystpruyst, 193 I, p. 55 ).

24. Cfr. L. Erba cit., p. I 33.

25. Cfr. ancora P. Tagliaferri cit., p. 74, in merito al rapporto che attraverso questi passi si delinea fra Du Bartas e Tasso.

26. Come sottolinea G. Jori, «nelle raffigurazioni astrali il Tasso ottenne alcuni dei risultati maggiori della nuova poetica di parafrasi scritturale » (Dal frammento al cosmo. Idoli e pietas dai "Dialoghi» al "Mondo Creato», in Torquato Tasso e la sua fortuna, Pisa-Roma, Istituti Editoriali e Poligrafici internazionali, I995, p. 405).

27. Ovvero Cosmologia di un traduttore, (in B. Basile cit, p. 175 e sgg.). Particolarmente utile al mio discorso si è poi rivelato il saggio di G. Baldassarri, Il Giolito, il Tasso, la Fenice. Un postillato disperso, in Studi in onore di Vittorio Zaccaria, a cura di M. Pecoraro, Milano, Unicopli, 1983, pp. 303-25.

28. B. Basile cit., pp. I 86-i 87. Un altro particolare curioso è ai vv. I56I e segg., dove il Tasso, rivolgendosi al sacro uccello, scrive : «O fortunato, e di te padre e figlio / Felice augello, e di te stesso erede, / Nutrito e nutritor, cui non distingue / Il vario sesso, e lunga età vetusta ». Questi versi sembrano trovare un rimando evidente, nonché un'efficace sintesi poetica nei seguenti sciolti guisoniani : «Ne la tomba infinito il proprio fine / Il rende, à se medesimo diventa / Per una cara aventurosa morte / Padre, madre, nutrice, herede, et figlio » (c. IO2V). 


\section{La Divina Settimana di Ferrante Guisone}

29. In realtà la creazione dell'uomo era già stata affrontata alla fine della lunga digressione sugli animali nel VI Giorno.

30. Cfr. P. Toldo, Due articoli letterari, Il poema della creazione di Du Bartas e quello di Torquato Tasso, Roma, Loescher et C., I 894.

3I. Cfr. ancora M. Tibaut de Masières cit., pp. 54-55. Una fonte comune è Ovidio : «os homini sublime dedit caelumque videre / iussit et erectos a sidera tollere vultus » (Metamorfosi, I, vv. 85-86).

32. Questo sintagma (che ritroveremo, fra l'altro, al v. ı०48 : «E l'uom creò, ch'è quasi un picciol mondo ") conosce una sua storia all'interno della poetica tassiana, come è testimoniato da un celebre passo del secondo dei Discorsi dell'arte poetica, Torquato Tasso, Discorsi dell'Arte poetica e del poemo eroico, a cura di L. Poma, Bari, Laterza, I964, p. 36 : «così parimente giudico che da eccellente poeta (il quale non per altro divino è detto se non perchè, al supremo Artefice nelle sue operazioni assomigliandosi, della sua divinità viene a partecipare) un poema formar si possa nel quale, quasi in un picciolo mondo, qui si leggono ordinanze d'esserciti, qui battaglie terrestri e navali, qui espugnazioni di città, scaramucce e duelli, qui giostre, qui descrizioni di fame e di sete, qui tempeste, qui incendii, qui prodigii ».

33. In merito alla creazione di Eva, si legga l'efficace panoramica offertaci da M. Hugues nel suo studio intitolato Le sommeil d'Adam et la création d'Eve dans la littérature bexamérale des $X V I^{e}$ et XVII siècles, in « Revue de littérature comparée», 2, I975, pp. 179-203.

34. Il poema del Du Bartas si avvalse di una straordinaria fortuna editoriale : l'edizione del I 578 fu la prima di una serie di ristampe, cui si aggiungono le numerose traduzioni in tutte le lingue europee (Ma vedi in proposito la nota di G. Jori cit., pp. 8-9). Anche la versione del Guisone conobbe una discreta diffusione, dal momento che nel i 6o la Divina Settimana era arrivata alla sua quinta edizione. 